\title{
Enhancement of Energy Aware Hierarchical Cluster-based Routing Protocol for WSNs
}

\author{
Er. Simranpreet kaur \\ M.Tech Scholar Department of Computer Science \& Engineering, Amritsar College of engineering and technology, \\ Amritsar, India \\ Email: Simran.bhullar113@gmail.com \\ Er. Shivani Sharma \\ Associate Professor, Department of Computer Science \& Engineering, Amritsar College of engineering and technology, \\ Amritsar, India \\ Email: er.sharma04@gmail.com
}

Received: 08 February 2018; Accepted: 08 March 2018; Published: 08 April 2018

\begin{abstract}
Wireless sensor networks are present almost everywhere because of their extensive variety of utilization. However, sensor nodes are battery constrained. Therefore, proficient utilization of power turns into testing issues. Aggregated data at the base station, by individual nodes cause a flood of information which results in greater power consumption. To avoid or minimize this issue a new technique of data aggregation has been proposed. In this paper, we proposed enhanced novel energy aware hierarchical cluster-based (ENEAHC) routing protocol with the aim to: minimizing as much as total energy consumption and to enhance the performance of the energy efficient protocol by using inter-cluster based data aggregation. LZW based data aggregation likewise connected to the Cluster head $(\mathrm{CH})$ to improve more results. Performance results show ENEAHC scheme reduce the end-to-end energy consumption and prolong the lifetime of the network compared to well known clustering algorithms i.e. LEACH and NEAHC. We design the actual relay node selecting issue like a non-linear programming issue and make use of property of compress sensing to find the optimal solution. The results are evaluated at the end of this paper through simulation.
\end{abstract}

Index Terms-LZW compression, LEACH, NEAHC, ENEAHC, Data Aggregation.

\section{INTRODUCTION}

Wireless sensor networks (WSNs) due to an assortment of uses [1-3] and future potential [4,5] picked up an enormous consideration among the scientists. Two primary segments of a WSNs are so-called as the sink node and sensor nodes. Common sensor nodes can complete detecting, information handling, what's more, conveying parts, making it attainable for an extensive variety of promising applications. Moreover, sensor nodes are typically battery-fueled and left alone make it very difficult to energize its nodes batteries or its replacement. Henceforth, one of the imperative issues in WSN is the manner by which to prolong the lifetime of the network with compelled energy asset. In spite of the way wireless sensor networks are fit for having a variety of network topologies, for example ring, star or mesh, the signs created by the nodes are towards the end clients through the sink nodes. A sink node or a base station is essentially an assigned gadget like typical sensor nodes however more intense. One of the essential tasks of sink node is to associate a WSN with its remote clients (Fig. 1). As an ad hoc network, the matter of fact is not same, as specially appointed systems, sensor nodes in WSN are controlled by non-rechargeable batteries [5]. Therefore the strategies [6-8] and outline of the novel convention $[9,10]$ to drag out the life span of the system are of big import. The power necessary to highway a message to the sink node, for each last node in sensor field that relies upon the separation from the sink node and figure nodes that the message should move. Having few sink nodes, employed efficiently in system field would decrease the power required to be convey a message and drag out the lifetime of the system. However, there are some limitation utilizing few sink nodes, for example the cost of the gadget or not being useful, to have more than one in the field. Because of the way that, the sensed information gathered by the sensor nodes is exchanged to the sink node, the whole execution can be affected through the position of the sink node.

Faced few difficulites in sequence to find an ideal stain of the sink node in the system field. May be a couple of fundamental issues are as follows:

1. There exists an enormous arranged space which implies the sink node can be situated in any place in the system field.

2. With the huge number of nodes in the field of sensor network are further fundamental test during finding sink node. 
3. There are distinctive routing protocols, each one having its own energy form and method to improve and transmit information toward the sink node.

4. Conceivable changes inside the system topology because of any kind of failure or up grader which may involve the sink node to be moved.

5. Enhancement of sink node area for various examining modes, for example, periodical or occasion-driven, might require diverse consideration [11].

6. Addition of sensor node in the system field requires the sink node to be repositioned so as to enhance the lifetime and also the throughput of the system.

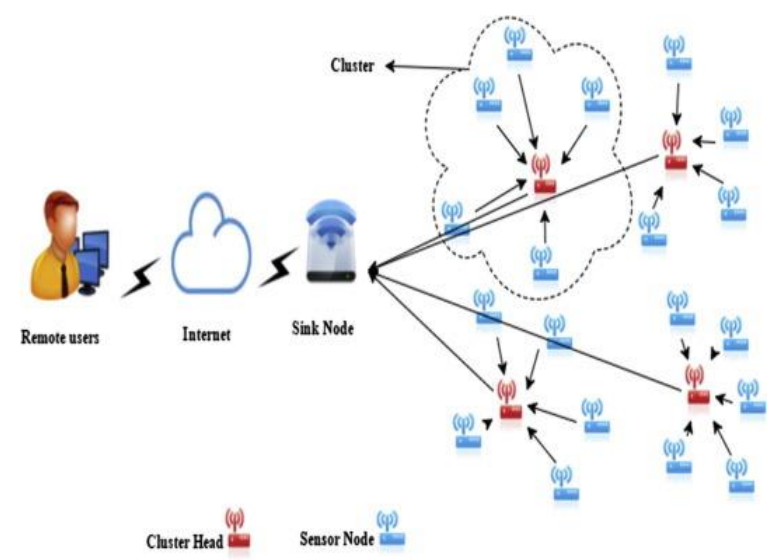

Fig.1. A usual wireless network

In particular, in this paper, we propose another routing protocol for wireless sensor networks (WSNs) called as ENEAHC to expand the lifetime of the network using a combination of compress sensing approach and an artificial bee colony (ABC) algorithm for optimal path selection. The proposition is to decide an ideal directing way from the source to the goal by favoring the most elevated battery power, least energy utilization in the multi-hop way, and ideal among sensor nodes. Hence, simulation results demostrate that this routing protocol not only adjusts the energy utilization of genernal system, it delays the dead time of sensor nodes, yet in addition gives more reliable delivery rate of data packets for transmission.

The rest of the paper is planned as follows. In Section 2 , we proceed with related work. Section 3, describe a selection of CHs. In Section 4, we proposed new protocol (ENEAHC). In Section 5, shows experimental setup and results. The performance evaluation described in Section 6 and at last we give conclusions and future scope in Section 7.

\section{RELATED WORK}

Many different approaches have been taken out to increase the energy efficiency of the network by applying clustering techniques in routing protocol [7-12].
Heinzelman et al. [7] Has been proposed that single level clustering based algorithm, called as LEACH (i.e. Low-Energy adaptive clustering hierarchy). It assumes the communication of sensor nodes with base station directly. LEACH chooses some part from all sensor nodes to serve as cluster heads with the objective to serve energy. The remaining sensor nodes become a part of the proper cluster in accordance to the signal strength from cluster heads. This procedure is isolated into various stages, each stage has a group set up stage to form clusters, and a rest stage in which the cluster heads ( $\mathrm{CHs}$ ) gather the information which they receive from their group individuals and after that the base station gets this gathered information from single node communication. After the completion of one phase, new coordinates are selected. The selection is made on the basis of random selection of a node from the nodes that are not selected yet as a coordinator or the coordinator is selected minimum number of times.

There are a few points of interest of utilizing $\mathrm{LEACH}$ algorithm for cluster formation, however, there is no assurance about the situation as well as no of group head nodes. The overall performance is not much affected during a particular phase as the clusters are adaptive in accordance to a poor clustering setup. The best outcomes are created by scattering the cluster head nodes all through the system utilizing a focal control algorithm. This is the idea behind the (LEACH-C) LEACHcentralized protocol, [8] a protocol which uses centralized clustering algorithm otherwise the same function is followed as followed by LEACH. As per this convention the information from the sensor nodes about their present area and energy level is received by the base station and afterward the transmission schedule is sent by the cluster head.

LEACH-C is better than the LEACH as the whole picture of the topology for selection of cluster heads and hence saves the energy of sensor nodes.

LEACH and LEACH-C although still standing up in the technology till now, they still can be introduced to enhancements in lifetime and other aspects. LEACH and LEACH-C does not consider different modes of functionality of nodes, such as the modes considered in this paper which switch modes from functioning to sleeping modes depending on an intelligent analysis of data.

In [9], proposed LEACH-CE protocol that only $5 \%$ of the total cluster heads are alive nodes. Just $10 \%$ of the nodes in the system let rest mode by the base station and this is done before the choice of the group heads. In the base station, nodes which are in rest mode don't detect any information nor the header data have got by them from the base station. Operation of all nodes is controlled by the base station. The base station selects only $5 \%$ of the cluster heads and the cluster heads selected in the previous phase are not selected again and again and this process continued till all the nodes in the network became cluster heads. This model randomly chooses the nodes which are switched to sleeping mode and does not 
guarantee the quality of data, although the data is preserved for a lifetime.

In [5], the proposed routing scheme utilizes node planning for each group in the system. The structure of the proposed scheme is an indistinguishable technique from within the typical LEACH convention. Calculating the number of sensor nodes, the arrangement of the clusters and furthermore the group head choice is the same in this proposed convention as LEACH. By doing this system more than once, the aggregate energy proficiency expanded as a result of the correct node scheduling inside the group. In this node scheduling, the aggregate accessible residual energy is similarly conveyed, and the group head is likewise chosen according to the remaining energy correlation. At whatever point the node is in rest mode in each group, every small energy is being consumed. Here, the aggregate rounds are expanded by doing both changing of resting and active modes and the accessible energy is appropriated in an adjusted way. Although this protocol does not choose the sleeping nodes randomly, it does not put into consideration the quality of sent data.

In [10], a protocol had been proposed which also switches a percentage of nodes into the sleeping mode using three factors which are; the energy level, the number of neighbors, and the average distance between neighbors. Clients that have a lot of neighbors and have a high energy level are good prospects to be switched to sleeping mode, as the more the number of neighbors of a node is the more overlap it has, and also the lower the energy the more probable it is to fail.

Wagh et.al [5] proposed the actual wifi sensor / probe systems are built to mount the actual clever circle software or even circle pertaining to unexpected emergency solutions, wherever our relationship is not possible. The nodes around wifi sensor / probe systems have to home coordinate according to needs by overseeing environments. Hence giving her very troubles to develop the actual sensor / probe circle for a max time period time period of the circle as well as a low power function with the nodes.

\section{Selection of Cluster Head}

LEACH performs its operations in rounds. Suppose that during each and every round, there are $\mathrm{k}$ numbers of clusters. In case sensor nodes begin with the similar energy, the main aim is to allocate the power load equally among all the nodes present in the sensor network. This is done so that no extremely utilize sensor nodes are present which will terminate of power before the other nodes. Initially by the starting of round $\mathrm{r}+1$ that begins with instant $\mathrm{t}$, with the probability $P_{S}(\mathrm{t})$ every node elects itself to become CHs (cluster head). $P_{s}(\mathrm{t})$ is selected such that for this round, $\mathrm{k}$ number of cluster heads are estimated. So, if $\mathbf{M}$ number of nodes in the sensor network are present,

$$
\mathrm{E}[\# \mathrm{CH}]==\sum_{S=1}^{M} P_{S}(\mathrm{t}) * 1=\mathrm{k}
$$

It must be assured that all the sensor nodes become $\mathrm{CHs}$ the equal number of times. Along with an average of $\mathrm{M} / \mathrm{k}$ rounds, it requires every last node to become a $\mathrm{CH}$ exactly once with their probabilities by using formula written below,

$$
P_{S}(\mathrm{t})=\left\{\begin{array}{c}
\frac{k}{M-k *\left(\operatorname{rmod} \frac{N}{k}\right)}: H_{S}(t)=1 \\
0: H_{S}(t)=0
\end{array}\right.
$$

If $H_{s}(t)=1$, after that nodes haven't become a $\mathrm{CH}$ within the current $r \bmod (\mathrm{M} / \mathrm{k})$ rounds. In the case of $H_{s}(t)=0$, nodes become a $\mathrm{CH}$.

$(M-k * r)$ Is the expected number of sensor nodes, which were not $\mathrm{CHs}$ in the initial $\mathrm{r}$ rounds. It is required that all nodes have been a cluster head after $\mathrm{M} / \mathrm{k}$ rounds once and they can do this job in coming that series of rounds. The expression $\sum_{s=1}^{M} H_{s}(t)$ Indicates the eligible nodes to become a $\mathrm{CH}$ at time $\mathrm{t}$.

$$
\mathrm{E}\left[\sum_{s=1}^{M} H_{s}(t)\right]=\left(M-k *\left(\operatorname{rmod} \frac{M}{k}\right)\right)
$$

After every N/k round, at all nodes the energy is roughly equivalent to one another. With the help of (2) and (3), the amount of $\mathrm{CHs}$ expected each round to be

$$
\begin{gathered}
\mathrm{E}[\# \mathrm{CH}]=\sum_{S=1}^{M} P_{S}(\mathrm{t}) * 1 \\
\sum_{s=1}^{M} P_{S}(\mathrm{t}) * 1=\left(M-k *\left(\operatorname{rmod} \frac{M}{k}\right)\right) * \frac{k}{M-k *\left(\operatorname{rmod} \frac{M}{k}\right)}=\mathrm{k}
\end{gathered}
$$

This probability to get a cluster head requires that all sensor nodes begin with same quantity of power and have information to broadcast during each round. In case it is assumed that all sensor nodes have the dissimilar amount of power, then those nodes having higher power than others should become cluster heads more number of times. To accomplish this, the probability to become a cluster head is set as a function of power level of a sensor node relative to the aggregate power left behind in the sensor network. So,

$$
P_{S}(\mathrm{t})=\min \left\{\frac{E_{S}(t)}{E_{\text {total }}(t)} k, 1\right\}
$$

Where $E_{S}(t)=$ the current power of nodes and

$$
E_{\text {total }}(t)=\sum_{s=1}^{M} E_{s}(t)
$$

With the aid of these probabilities, the higher power nodes will become cluster heads than lower power modes. The amount of cluster head nodes expected is

$$
\mathrm{E}[\# \mathrm{CH}]=\sum_{s=1}^{M} P_{S}(\mathrm{t}) * 1=\left(\frac{E_{1}(t)}{E_{\text {total }}}+\cdots \ldots \ldots \ldots+\frac{E_{N}(t)}{E_{\text {total }}}\right) \mathrm{k}=\mathrm{k}
$$

When the sensor nodes start with the same energy, the equation (5) is approximated by (2). 


\section{A. Algorithm for cluster formation}

After the nodes electing themselves as cluster heads with the help of (4) or (7), the cluster heads should tell other sensor nodes in the network that they have been selected as a leader for the present round. An advertisement message is broadcasted by each cluster head node which includes the ID of the node and a header which differentiates it as a message of the annunciation. On the basis of the least communication power or received signal strength from each and every cluster head, sensor nodes select its cluster for the current round. More signal strength means the minimum amount to transmit power to interact with. Cluster head is taken randomly during ties. Deciding which cluster, each node belongs to, cluster head is informed about its cluster member nodes. A request message is generated which include node ID and the CH ID is sent by sensor nodes back to the selected group heads. A TDMA schedule is set up by cluster head and this is sent to the sensor nodes present in the cluster, which is used to ensure that no collisions take place between the data messages and the radio components of each and every cluster member nodes are allowed to be turned off at all times except they're spending time. It facilitates in cutting down the power usage by individual sensor nodes. Later on the nodes knowing this schedule, the setup phase is completed and the steady phase can begin.

\section{B. Steady phase}

Here, the operation is divided into some frames. During the assigned slot of transmission, data are transmitted by sensor nodes to the cluster head exactly once per frame. The period of each and every slot in which data is carried by a sensor node is constant, therefore the time for sending data frames depends on the number of sensor nodes present in the cluster. It is assumed that the time synchronized nodes begin the setup state at the similar instant. On the base of the received signal intensity from the advertisement of the cluster head, for reducing power dissipation, power control is used by each sensor node to set the quantity of sending power. Turn off the radio of sensor nodes until their assigned time of transmission. For receiving all the information from the sensor nodes present in the cluster, it is necessary for the CHS to be awake. For enhancing the common signal and reducing noise among the signals, data aggregation is performed by cluster head on receiving data. The cluster head transmits the consolidated information to the base station. During transmission, high energy is consumed because of the large size of the data and position of base station may be far off.

Communication in close by cluster will be affected by transmission occurring in one cluster. Using directsequence spread spectrum (DSSS), communication is performed in LEACH by each and every cluster for reducing inter-cluster interference. A unique spreading code is used by each cluster. By using this spreading code, data are sent to $\mathrm{CH}$ by all the sensor nodes present in the cluster and all received power is filtered by the cluster head using the same code. On a predefined list, for advertising the position, the first cluster head is assigned the first code, the second head the second code, so on. Transmit energy is set by each node for minimizing the chances of interfering with close by clusters and minimizing its own power. For ensuring a low collision probability, there is a need of few transmissions that are overlapping and slight data spreading. With the help of a fixed spreading code and CSMA, the cluster head nodes transmit data to the base station. If a cluster head has information at the end of its frame to transmit, it first checks whether any other cluster head is sent using the spreading code of base station or not. If yes, then the cluster head will have to wait. Or else, the data are sent to the base station by cluster head using the spreading code of the base station. For performing data aggregation for compressing information into a single signal at cluster head, information from distinct sensor nodes in the cluster must be interrelated. Suppose a distance $\rho$ is traveled by a source signal before it cannot be detected by sensor nodes. The maximum distance between sensor nodes having similar information is $2 \rho$. Within this distance, it cannot be guaranteed that the same signal will be detected by the two sensor nodes. In the event that all the sensor nodes are in a group having distance across d (i.e. Most extreme separation between two sensor nodes is d), and the estimation of $\mathrm{d}<2 \rho$, the perspectives of individual sensor nodes will overlap. In such a case, the data from distinct sensors will correlate.

\section{Data aggregation functions}

If $A_{i}$ And $B_{j}$ Are two variables that represent the correlation [20] of information in packets that are generated by the nodes ' $\mathrm{N}$ ' taking part in the cluster, where $i=1 . . X$ and $j=1 \ldots Y$, then the aggregation function will be given by:

1. Addition function: If packet generated by each node has different information, then the resulting function will be the addition of packets from all nodes, taking part:

$$
\text { Addition }=\sum_{i=1}^{X}\left(A_{i}\right) \text { For } \forall\left(A_{i}\right)=\text { Distinct Data }
$$

2. Divisible function: If each node generates packets having similar data, then the result will be shown as:

$$
\text { Division }=\frac{1}{Y} \sum_{j=1}^{Y}\left(B_{j}\right) \text { For } \forall\left(B_{J}\right)=\text { Similar Data }
$$

Where $\mathrm{Y}=$ nodes having similar data packets

\section{Proposed Protocol (ENeAhC)}

To simulate the protocol, we modified some additional parameters and some existing equations . Subsequent 
section contains various parameters to simulate the desired requirements.

Maximum compression ratio of $\mathrm{LZW}=9.42$

Compressed packet size (CPS) is defined as

$$
C P S=\frac{A P S}{9.42}
$$

Where APS is the actual packet size.

Therefore new energy dissipation for compressed data will be as:

$$
\begin{aligned}
S(i) \cdot E=S(i) \cdot E & -\left(\left(T x_{\text {energy }}+E D A\right) * C P S+A M P\right. \\
& * C P S * d^{4}: \text { ifd }>d_{o} \\
S(i) \cdot E=S(i) \cdot E & -\left(\left(T x_{\text {energy }}+E D A\right) * C P S+E M P\right. \\
& * C P S * d^{2}: \text { ifd }<d_{o}
\end{aligned}
$$

/*Initialization of WSN*/

1. Deploy nodes randomly based upon normal distribution

/*Setup phase*/

2. Select cluster head using threshold function $P_{S}(\mathrm{t})$. It is evaluated as follows:

$P_{S}(\mathrm{t})=\left\{\begin{array}{c}\frac{k}{M-k *\left(r \bmod \frac{M}{k}\right)}: H_{S}(t)=1 \\ 0: H_{S}(t)=0\end{array}\right.$

Here, "M" represents sensor nodes, "K" are the approximate number of cluster heads, " $r$ " is current round. It will be evaluated if $H_{s}(t)=1$.

3. Elected cluster head $(\mathrm{CH})$ to communicate message to member nodes.

4. TDMA(Time Division Multiple Address)will be used communicate data with sink.

5. Apply inter cluster data aggregation on root $C H$ using $A B C$

6. Root $\mathrm{CH}$ transmit data to sink by applying compressive sensing on aggregated packet.

7. Evaluate and update remaing energy as follows:

$S(i) \cdot E=S(i) \cdot E-\left(\left(T x_{\text {energy }}+E D A\right) * C P S+A M P\right.$

$$
* C P S * d^{4}: \text { ifd }>d_{o}
$$

$S(i) \cdot E=S(i) \cdot E-\left(\left(T x_{\text {energy }}+E D A\right) * C P S+E M P * C P S\right.$ $* d^{2}:$ ifd $<d_{o}$

8. Check dead nodes as follows:

9. Countdead nodes

10. Check termination criteria as follows:

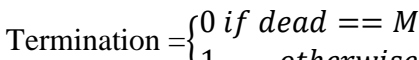

11. if dead $==M$

12. Return network lifetime

13. Else

14. Move to Step 2.

15. End of code.

In above equations, $\mathrm{S}(\mathrm{i})$. $\mathrm{E}$ indicates the energy of $\mathrm{i}^{\text {th }}$ node, Transmitter energy is denoted by symbol T $x_{\text {energy }}$, EDA is effective data aggregation cost, AMP is channel cost of multipath fading and EMP is channel cost of free space.

The distance between $\mathrm{CHs}$ and BS is denoted by symbol ' $\mathrm{d}$ ' and $d_{o}$ is the min. distance.
The proposed calculation will work in following stages i.e. Cluster arrangement (Selection of cluster head) intra-cluster aggregation (grouping of nodes for transmission of parcels to cluster sets out toward aggregation), between cluster aggregation(grouping of Cluster sets out toward transmission of accumulated information to the transfer node) and data compression.

\section{A. Algorithm for modified leach}

\section{EXPERIMENTAL SET-UP}

The proposed algorithm has been designed and executed in MATLAB using the data analysis toolkit. Subsequent section contains the result of the leach protocol.

Below are some symbols to describe the sensor field in proposed network.

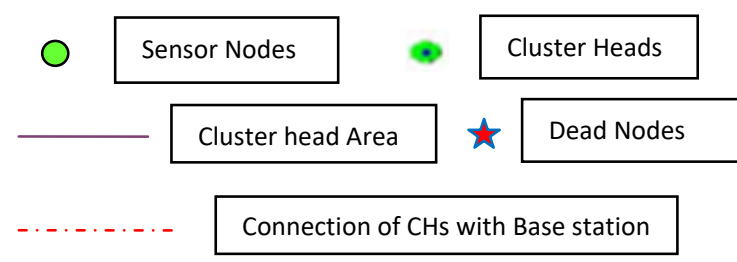

On applying ENEAHC protocol, the following outcomes were assembled. In view of these outcomes, a detailed analysis is presented. For analyzing the performance of ENEAHC protocol, randomly 100 sensor nodes are sent in a geological area A with measurement 100 by $100 \mathrm{~m}$. The accompanying Fig 2 demonstrates Advanced nodes where all nodes are alive, Fig 3 demonstrates some dead nodes after a couple of rounds.

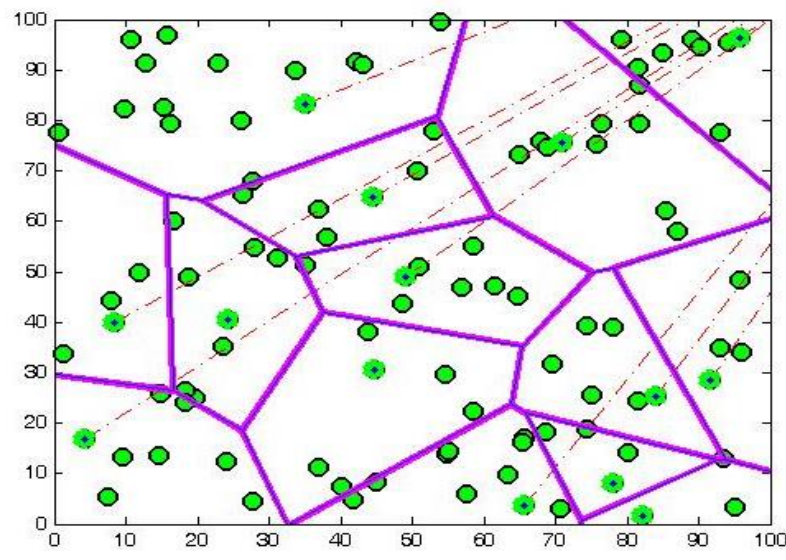

Fig.2. When all the sensor nodes are active

In Fig 2 showing the initial environment in which each sensor node has full of energy and versatile in nature 


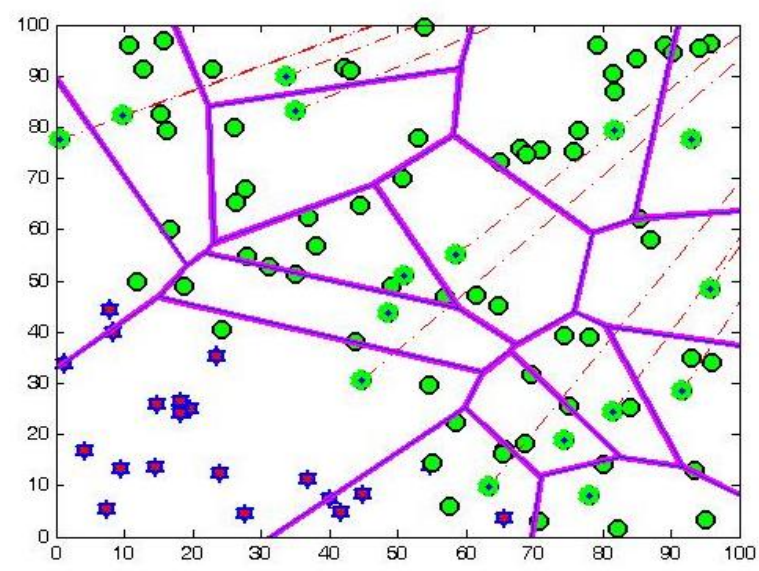

Fig.3. When some of the sensor nodes are dead

Fig 3 has indicate same environment of the protocol as shown in above. Red color stars are addressing regular sensor nodes, which are dead. When a node is known to be dead, if its energy become zero this means that the node is no longer available for communication.

\section{PeRformance Evaluation}

Subsequent section contains the comparison among our protocol i.e. ENEAHC with LEACH, NEAHC protocols. We consider it with following metrics:

\section{Network Lifetime \\ 2.Remaining Energy \\ 3. Data Packet Delivery}

Table 1 has demonstrated the different parameters required to effectively simulate the WSNs. The values taken are the benchmark for WSNs.

Table 1. Simulation Parameters

\begin{tabular}{cc}
\hline Parameter & Value \\
\hline WSN Area $\left(\mathrm{m}^{2}\right)$ & $100 * 100$ \\
Location of Base station & 150,150 \\
No. of Nodes & 100 \\
$P_{S}$ (probability) & 0.1 \\
Initial Energy & $0.1 \mathrm{~J}$ \\
Energy_transmiter & $50 * 10^{-9} \mathrm{~J} / \mathrm{bit}$ \\
Energy_receiver & $50 * 10^{-9} \mathrm{~J} / \mathrm{bit}$ \\
Free space(amplifier) & $10 * 10^{-13} \mathrm{~J} / \mathrm{bit} / \mathrm{m}^{2}$ \\
Multipath & $0.0013 * 10^{-13}$ \\
& $\mathrm{~J} / \mathrm{bit} / \mathrm{m}^{2}$ \\
Effective Data aggregation & $5 * 10^{-9} \mathrm{~J} / \mathrm{bit} / \mathrm{signal}$ \\
Max lifetime & 4000 \\
Size of Data packet & $4000 \mathrm{~KB}$ \\
LZW compression & 9.42 \\
\hline
\end{tabular}

When Numbers of nodes are 100:

First, we examine the network lifetime of LEACH, NEAHC and our protocol. We analyze the way that the quantity of alive nodes changes as the network develops.

In Fig.4, shows that proposed protocol has enhanced lifetime of the network that the other existing protocols. Use of mobile sink, it limited communication cost and direct information exchange by nodes near base station prolong the system lifetime.

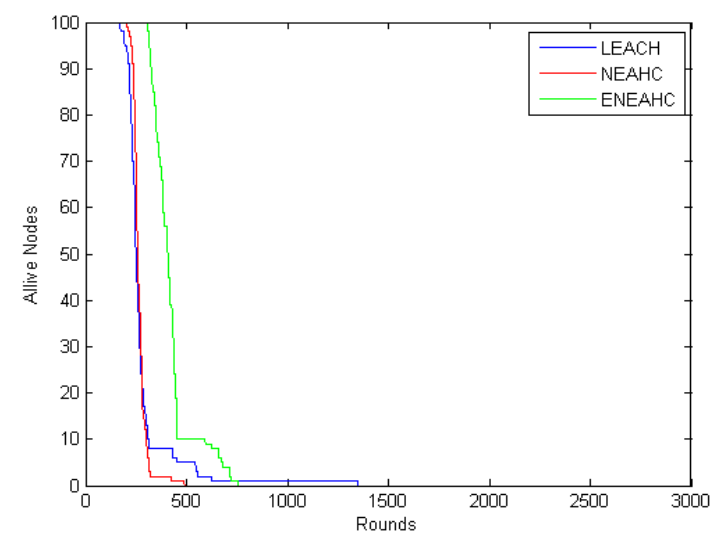

Fig.4. Alive Nodes for Network

In Fig.5, shows the data packet delivery to the sink nodes i.e. BS. For huge networks, $\mathrm{CHs}$ are not in the range of some other sensor nodes, in that case there are loss in data packets. Rate of data packet delivery is improved for the proposed protocol than LEACH and NEAHC.

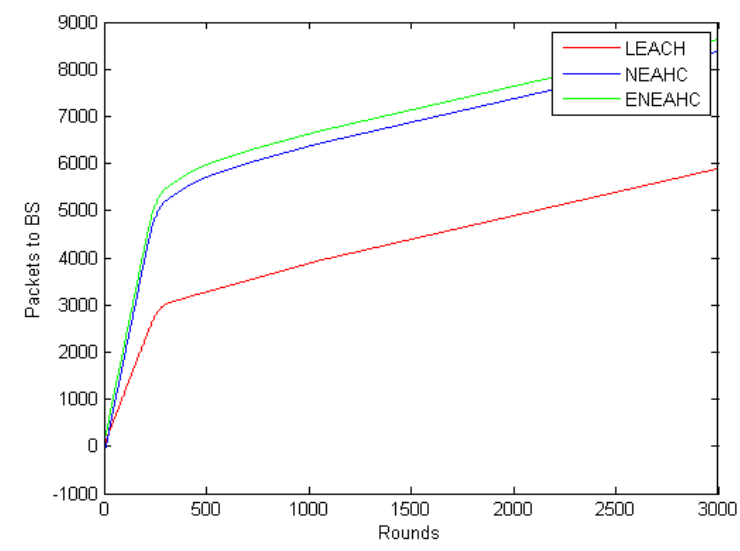

Fig.5. Data packet delivery for network

In Fig.6, shows less energy consumption in the proposed protocol as compare to LEACH and NEAHC.

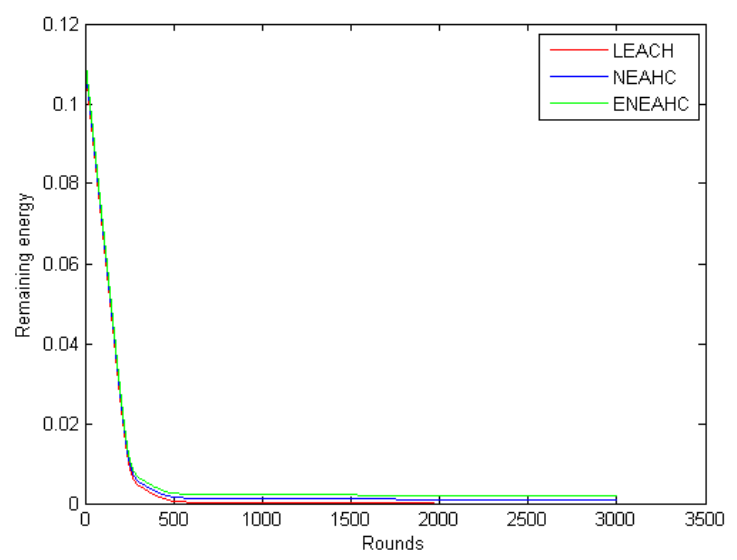

Fig.6. Remaining Energy of Network 
As the main objective of clustering is to maximize the first node and last node dead time. (Leach, $1^{\text {st }}$ dead-72, leach all dead-293, Daleach1st-182, da leach all-309, Lzw1st-196 . Lzw all-416)

Table 2 shows better results of proposed protocol ENEAHC than LEACH and NEAHC protocol in lifetime, stable period of nodes and mean packet delivery rate.

Table 2. Dead node evaluation

\begin{tabular}{|c|c|c|c|}
\hline $\begin{array}{c}\text { Protocols } \\
\mathbf{N = 1 0 0}\end{array}$ & Lifetime & $\begin{array}{c}\text { Stable } \\
\text { Period } \\
\text { Of } \\
\text { Nodes }\end{array}$ & $\begin{array}{c}\text { Mean } \\
\text { Packet } \\
\text { delivery } \\
\text { rate }\end{array}$ \\
\hline LEACH & 383 & 73 & 3.86 \\
\hline NEACH & 430 & 198 & 4.34 \\
\hline ENEAHC & 471 & 203 & 4.76 \\
\hline
\end{tabular}

\section{CONCLUSION AND Future DiRECTIONS}

Improving network lifetime is formed to be a calling issue in existing research on the energy efficient protocol of WSN's. It has been observed that the data aggregation, compress sensing and an efficient path selection technique can improve network lifetime. To achieve this, in this research work we have proposed an efficient compress sensing based on inter-cluster data aggregation techniques to reduce the packet size of sensor nodes. Additionally, ABC (Artificial Bee Colony) based path selection technique has been used to reduce the energy consumption will transmit the packets. Extensive analysis shows that the proposed technique out formed to well known energy efficient protocol, which are LEACH and NEAHC. It has been observed for comparative analysis that proposed has improved $1.2671 \%$ network lifetime that NEAHC protocol. Therefore, proposed is more efficient for real time WSN's.

However, in this work effect of security in WSN has been neglected. In this manner, sooner rather than later, we will extend the proposed strategy for security issues.

\section{REFERENCES}

[1] P. N. Renjith and E. Baburaj, "An Analysis on Data Aggregation in Wireless Sensor Networks," International Conference on Radar, Communication and Computing (ICRCC), SKP Engineering College, Tiruvannamalai, TN., India. pp. 62-71, Dec. 2012.

[2] I.Akyldiz, W.Su, Y.Sankarasubramanian and E. Cayirci, "Wireless Sensor networks: a survey," IEEE Computer, vol. 38, no. 4, pp. 393-422, 2002.

[3] R.Rajagopalan and P.Varshney, "Data-Aggregation Techniques in Sensor Networks: A Survey,” IEEE Comm. Surveys Tutorials, vol. 8 , no. 4, pp. 48-63, Oct.-Nov. 2006.

[4] S.Madden, M.J.Franklin, J.M.Hellerstein, and W.Hong, "TAG: A Tiny Aggregation Service for Ad-Hoc Sensor Networks," Proc. Fifth Symp. Operating Systems Design and Implementation, 2002.

[5] Ke, Wang, et al. "Energy aware hierarchical cluster-based routing protocol for WSNs." The Journal of China Universities of Posts and Telecommunications 23.4 (2016): 46-52.Izadi, Davood, JemalAbawajy, and Sara
Ghanavati. "An Alternative Clustering Scheme in WSN." (2015).

[6] S.Tilak, N.Abhu-Gazhaleh, W.R.Heinzelman, "A taxonomy of wireless micro-sensor network models," ACM SIGMOBILE Mobile Comp. Commun. Rev. , vol. 6, no. 2, pp. 28-36, Apr. 2002.

[7] I. Akyldiz, W.Su, Y. Sankarasubramanian and E. Cayirci, "A survey on sensor networks," IEEE CommunicationMag., vol. 40, no. 8, pp. 102-114, Aug. 2002.

[8] Olivier Dousse, PetteriMannersalo, Patrick Thiran, "Latency of Wireless Sensor Networks with Uncoordinated Power Saving Mechanisms," MobiHoc'04, May 24-26, 2004, Roppongi, Japan.

[9] HuseyinOzgur Tan and Ibrahim Korpeoglu, "Power Efficient Data Gathering and Aggregation in Wireless Sensor Networks".

[10] S. Cho, andA. Chandrakasan, "Energy-efficient protocols for low duty cycle wireless microsensor networks", Proceedings of the 33rd Annual Hawaii International Conference on SystemSciences, Maui, HI Vol. 2 (2000), p. 10.

[11] N. Bulusu, D. Estrin, L. Girod, J. Heidemann, "Scalable coordination for wireless sensor networks: selfconfiguring localization systems", International Symposium on Communication Theory and Applications (ISCTA 2001), Ambleside, UK, July 2001.

[12] C. Intanagonwiwat, R. Govindan, D. Estrin, "Directed diffusion: a scalable and robust communication paradigm for sensor networks" Proceedings of the ACM MobiCom'00, Boston, MA, 2000, pp. 56-67.

[13] S. Meguerdichian, F. Koushanfar, G. Qu, M. Potkonjak, "Exposure in wireless ad-hoc sensor networks", Proceedings of ACM MobiCom'01, Rome, Italy, 2001, pp. 139-150.

[14] W.Heinzelman, A.Chandrakasan, H.Balakrishan, "An application-specific protocol architecture for wireless microsensor networks", IEEE Transaction on Wireless Communication, vol. 1, no. 4, pp. 660-670, 2002.

[15] G. Smaragdakis, I. Matta, Bestavros, "SEP: A Stable Election Protocol for clustered heterogeneous wireless sensor network", in: Second international Workshop on sensor and Actor Network Protocols and Applications (SANPA 2004), 2004.

[16] O. Younis and S. Fahmy, "HEED: a Hybrid, EnergyEfficient Distributed Clustering Approach for Ad Hoc Sensor Networks", IEEE Trans. Mobile Computing, vol. 3, no. 4, Dec. 2004, pp. 366-79.

[17] Woo-Sung Jung, keun-Woo Lim, Young-BaeKo, SangJoon Park, "A hybrid approach for clustering-based Data Aggregation in Wireless Sensor Networks", IEEE, $3^{\text {rd }}$ International Conference on Digital Society, pp. 112-117, 2009.

[18] LingjunMeng, Huazhong Zhang, Yun Zou, "A Data Aggregation Transfer Protocol based on Clustering and Data Prediction in Wireless Sensor Networks", IEEE, 2011.

[19] JiPeng, Li Yupeng, Jiang Jingqi, Wang Tianbao, "A Clustering Protocol for Data Aggregation in Wireless Sensor Network", IEEE, International Conference on Control Engineering and Communications Technology, 2012.

[20] Siva Ranjani. S, Radha Krishnan. S, Thangaraj. C, "Energy- efficient cluster based data aggregation for wireless sensor networks", International Conference on Recent Advances in Computing and Software Systems, pp. 174-179, 2012. 
[21] DnyaneshwarMantri, Neeli R Prasad andRamjee Prasad, " Grouping of clusters for Efficient Data Aggregation (GCEDA) in wireless sensor network", IEEE, $3^{\text {rd }}$ International Advance Computing Conference, pp. 132137, 2013.

[22] DnyaneshwarMantri, Neeli R Prasad andRamjee Prasad, "BHCDA:Bandwidth Efficient heterogeneity aware cluster based data aggregation for wireless sensor network", IEEE, International Conference on Advances in Computing, Communications and Informatics, pp. 1064-1069, 2013.

[23] B. A. Said, E. Abdellah, A. B. Hussane and M. L. Hasnaoui, "Improved and Balanced LEACH for heterogeneous wireless sensor networks", International Journalon Computer Science and Engineering, Vol 02, No. 08, 2010, 2633-2640.

[24] M. Mittal, S. Srinivasan, M. Rani and O. P. Vyas, "Type2 fuzzy ontology-based multi-agents system for wireless sensor network," TENCON 2017 - 2017 IEEE Region 10 Conference, Penang, Malaysia, 2017, pp. 2864-2869.

[25] S. Gaikwad and M. Ghosal, "Energy efficient storage-less and converter-less renewable energy harvesting system using MPPT," 2017 2nd International Conference for Convergence in Technology (I2CT), Mumbai, India, 2017, pp. 971-973.

[26] M. Nikolov and Z. J. Haas, "Encoded Sensing for Energy Efficient Wireless Sensor Networks," in IEEE Sensors Journal, vol. 18, no. 2, pp. 875-889, Jan.15, 152018.

[27] R. Singh, B. K. Rai and S. K. Bose, "A contention based routing enhanced MAC protocol for transmission delay reduction in a multi-hop WSN," TENCON 2017 - 2017 IEEE Region 10 Conference, Penang, Malaysia, 2017, pp. 398-402.

[28] N. Sainath and D. Vasumathi, "A novel approach to identify the performance \& security in various routing protocols," 2017 International conference of Electronics, Communication and Aerospace Technology (ICECA), COIMBATORE, India, 2017, pp. 690-695.

[29] N. Zuriatunadhirah binti Zubir, A. F. Ramli and H. Basarudin, "Optimization of wireless sensor networks MAC protocols using machine learning; a survey," 2017 International Conference on Engineering Technology and Technopreneurship (ICE2T), Kuala Lumpur, Malaysia, 2017, pp. 1-5.

[30] K. Parashar and K. R. Ranjan, "Distributed data aggregation energy efficient cluster protocol based structural health monitoring," 2017 International conference of Electronics, Communication and Aerospace Technology (ICECA), COIMBATORE, India, 2017, pp. 518-522.

[31] H. I. Kobo, G. P. Hancke and A. M. Abu-Mahfouz, "Towards a distributed control system for software defined Wireless Sensor Networks," IECON 2017 - 43rd Annual Conference of the IEEE Industrial Electronics Society, Beijing, China, 2017, pp. 6125-6130.

[32] S. Mishra, J. Varavadekar and S. Haldankar, "Design of rectenna for energy harvesting in ISM band," 2017 International conference of Electronics, Communication and Aerospace Technology (ICECA), COIMBATORE, India, 2017, pp. 359-362.

[33] N. A. Ansari, U. A. Deshpande, A. M. Tapas and A. A. Jejani, "A distributed algorithm for object tracking in wireless sensor networks using data mining based predicition," 2017 8th International Conference on Computing, Communication and Networking Technologies (ICCCNT), Delhi, India, 2017, pp. 1-6.

[34] M. Narendran and P. Prakasam, "Optimized lowest ID in wireless sensor network using Invasive Weed Optimization (IWO)-genetic algorithm (GA)," 2017 International Conference on Algorithms, Methodology, Models and Applications in Emerging Technologies (ICAMMAET), CHENNAI, India, 2017, pp. 1-6.

[35] N. Sharma and K. Sharma, "Routing and clustering optimization techniques in WSN: A review," 2017 8th International Conference on Computing, Communication and Networking Technologies (ICCCNT), Delhi, India, 2017, pp. 1-7.

[36] M. Mihoubi, A. Rahmoun and P. Lorenz, "Metaheuristic RSSI based for node localization in distributed wireless sensor network," 2017 Global Information Infrastructure and Networking Symposium (GIIS), Saint Pierre, France, 2017, pp. 64-70.

[37] S. A. R. T. Heydariyan and A. H. Mohajerzadeh, "Using the combination of particle swarm algorithms and fuzzy approach to provide a clustering method for network nodes with coverage maintenance in wireless sensor networks," 2017 7th International Conference on Computer and Knowledge Engineering (ICCKE), Mashhad, Iran, 2017, pp. 20-26.

[38] N. Ajaykumar and M. Sarvagya, "Secure and energy efficient routing protocol in wireless sensor network: A survey," 2017 International Conference on Advances in Computing, Communications and Informatics (ICACCI), Udupi, 2017, pp. 2313-2322.

[39] G. Ge, "The application of the wireless sensor network on port wharf structure health monitoring," 2017 IEEE 3rd Information Technology and Mechatronics Engineering Conference (ITOEC), Chongqing, 2017, pp. 701-707.

\section{Authors' Profiles}

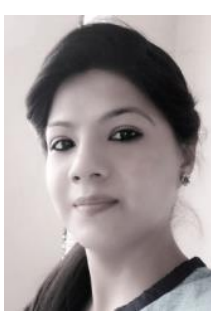

fog computing.
Simranpreet kaur received the B.Tech degree in computer science and engineering from Amritsar college of Engineering and Technology(ACET), Amritsar, India. She is currently pursuing the M.Tech degree with the department of computer science, ACET college, under supervision of Shivani Sharma. Her current research interests include wireless sensor networks, IOT and

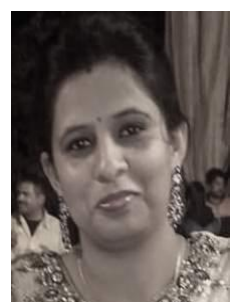

Shivani Sharma currently a Associate Professor in Amritsar College Of Engineering and Technology(ACET), Amritsar, India. She received B.Tech and M.tech Degree in computer science. Her current research interests include Knowlegde discovery in Sensor networks, espically clustering and computing. 
How to cite this paper: Er. Simranpreet kaur, Er. Shivani Sharma, " Enhancement of Energy Aware Hierarchical Cluster-based Routing Protocol for WSNs", International Journal of Modern Education and Computer Science(IJMECS), Vol.10, No.4, pp. 26-34, 2018.DOI: 10.5815/ijmecs.2018.04.04 